\title{
Ocean Dynamics and the Oxygenation of Habitable Worlds
}

\section{STEPHANIE OLSON}

Purdue University

Presenting Author: stephanieolson@purdue.edu

The origin of oxygenic photosynthesis did not immediately oxygenate Earth's atmosphere. By some estimates, oxygenic photosynthesis and low-level oxygenation of the shallow ocean preceded atmospheric oxygenation by several hundred million years. This temporal disconnect illustrates that biological oxygen production need not manifest as an oxygenated atmosphere on other inhabited worlds--and exoplanetary atmospheres may ultimately provide misleading windows to surface habitats.

This talk will explore how various planetary parameters--such as orbital obliquity, rotation rate, surface pressure, and continentality--affect biospheric oxygenation on early Earth and beyond. We focus in particular on sea-to-air oxygen fluxes and the possibility of sustained "oxygen oases" in disequilibrium with an anoxic atmosphere using 3D models for ocean dynamics and marine biogeochemistry.

We find that increasing day length, higher surface pressure, and the emergence of continents all influence ocean circulation patterns and associated nutrient transport in ways that may enhance biospheric oxygen production. We argue that these relationships may have contributed to Earth's oxygenation by favoring a secular increase in biogenic oxygen fluxes to the atmosphere. We additionally find that high orbital obliquity may be associated with greater oxygen fluxes to the atmosphere on annual average due to seasonal deepening of the mixed layer and resulting nutrient entrainment. Worlds that are tilted on their axes may thus be more likely to evolve complex life. 